\title{
Partial Anomalous Pulmonary Venous Return Diagnosed by Central Catheter Misplacement
}

\author{
Lena Reichert ${ }^{1}$ Ioannis Bougioukas ${ }^{1}$ Ralf Seipelt ${ }^{1}$ Hanno Huwer ${ }^{1}$ \\ ${ }^{1}$ Department of Cardiothoracic Surgery, SHG-Kliniken Voelklingen, \\ Voelklingen, Germany \\ Thorac Cardiovasc Surg Rep 2020;9:e37-e39.

\begin{abstract}
Address for correspondence Dr. Med. Ioannis Bougioukas, MD, Richardstr, 5-9, 66333, Voelklingen, 66333, Germany (e-mail: ybougioukas@yahoo.com).
\end{abstract} \\ Department of Cardiothoracic Surgery, SHG-Kliniken Voelklingen,
}

\begin{abstract}
Keyword

- pulmonary veins

- surgery

- cardiac anatomy

Anomalous venous connections of the left lung can either affect all of the veins or only the upper lobe. They mostly drain into the innominate vein. We present the case of a patient who underwent a coronary bypass operation and was prepared with insertion of central lines including Swan-Ganz catheter through both the internal jugular veins. Blood gas analysis obtained from these catheters suggested the presence of a left-toright shunt. CT (computed tomography) imaging confirmed a pulmonary venous anomaly with misplacement of the left-sided catheter in an abnormal pulmonary vein. Such a rare condition can be suspected by obtaining arterialized blood samples and measuring the mean pressure through central catheters.
\end{abstract}

\section{Introduction}

Anomalous pulmonary venous connections are a group of rare vascular malformations in which one or more of the pulmonary veins are connected directly to the right atrium or the systemic venous system, causing a left-to-right shunt. ${ }^{1}$ They can be either partial or total. They are encountered in approximately $0.5 \%$ of the pediatric population, whereas they are very rare in the adult population posing often an incidental finding during a cardiothoracic operation. ${ }^{2}$

\section{Case Presentation}

A 69-year-old female Caucasian patient with two-vessel coronary disease and unstable angina pectoris was admitted at our department for surgical myocardial revascularization. Central venous lines were inserted through both the internal jugular veins for monitoring and perioperative management.

Surgery was completed without any complications. Chest Xray at the intensive care unit ( - Fig. 1 ) showed a correct position of the right-sided Swan-Ganz catheter and a correct position of the left-sided central venous catheter under the assumption of the presence of a persistent left superior vena cava.

The mixed venous oxygen saturation of $68 \%$ was significantly lower than the central venous oxygen saturation of

received

May 1, 2020

accepted after revision

May 27, 2020
78\%. It was also possible to aspirate arterial blood with an oxygen saturation of $100 \%$ through the left-sided venous catheter. The central venous pressures, measured over the right- and left-sided venous catheters, were almost equal (11 and $17 \mathrm{~mm} \mathrm{Hg}$, respectively). All these led to the conclusion of the existence of a left-to-right shunt. A previous computed tomography (CT) of the thorax showed an until then not detected pulmonary vein arising from the left upper lobe and draining into the innominate vein (-Fig. 2). As a secondary finding, an aberrant subclavian artery was also found ( - Figs. 3 and 4). The left-to-right shunt was calculated according to the following formula: Qp / $\mathrm{Qs}=\left(\mathrm{SaO}_{2}-\mathrm{SvO}_{2}\right) /\left(\mathrm{SpvO}_{2}-\right.$ $\mathrm{SpaO}_{2}$ ), where $\mathrm{Qp}=$ pulmonary blood flow, $\mathrm{Qs}=$ systemic blood flow, $\mathrm{SaO}_{2}=$ systemic arterial oxygen saturation, $\mathrm{SvO}_{2}=$ central venous oxygen saturation, $\mathrm{SpvO}_{2}=$ pulmonary venous oxygen saturation, and $\mathrm{SpaO}_{2}=$ pulmonary arterial oxygen saturation; the result was $1.36 / 1$. The left central venous catheter was finally removed without any complications.

\section{Discussion}

Central venous catheters that are displayed on the X-rays to be lying in the left thoracic side can be located in various
License terms

Stuttgart · New York

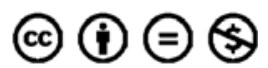




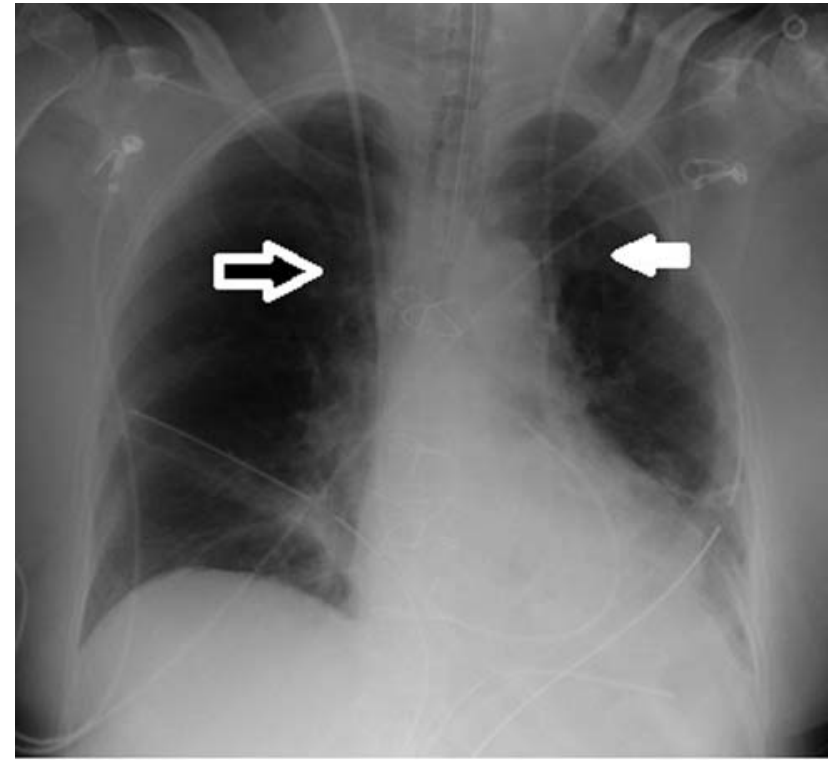

Fig. 1 Postoperative chest X-ray of the patient after myocardial revascularization through median sternotomy. The white arrow depicts the left-sided central venous catheter, and the black and white arrow shows the right-sided Swan-Ganz catheter.

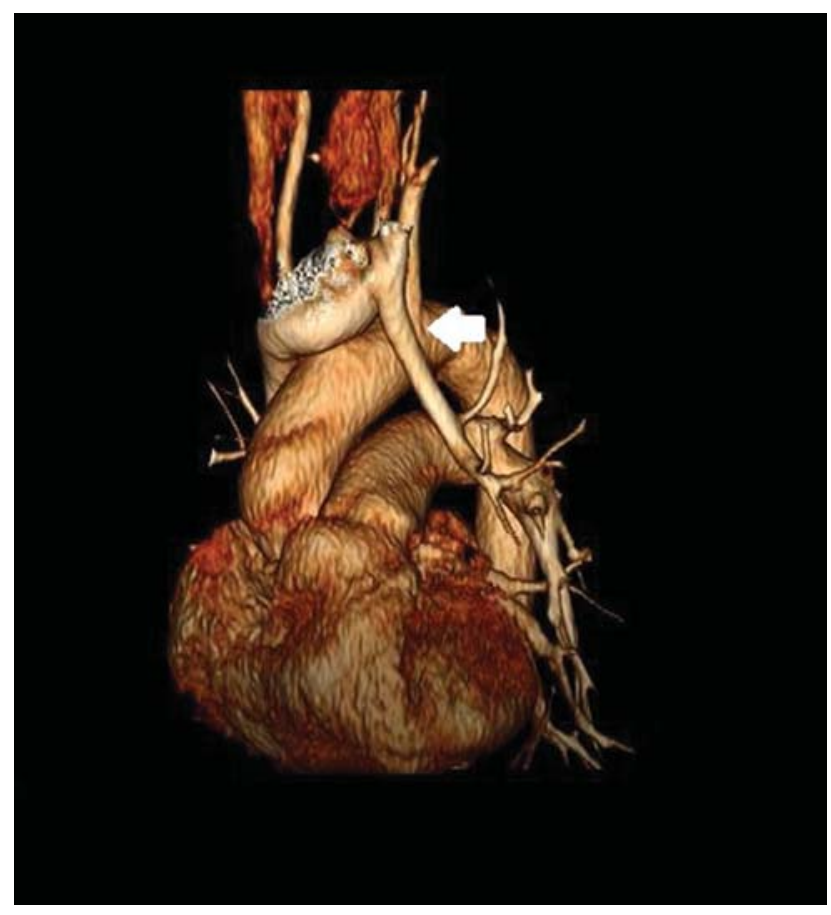

Fig. 2 Computed tomography of the thorax: three-dimensional reconstruction. The white arrow shows the anomalous pulmonary vein of the left upper lobe of the lung draining into the innominate vein.

blood vessels, provided they are located intravascularly. Such a catheter can be located in a left persistent superior vena cava that drains into the right atrium through the coronary sinus. This is the most common innate abnormal variant of the thoracic venous system with a prevalence of approximately $0.5 \%$ within the population. ${ }^{3}$ Because of the fact that the left persistent vena cava finally drains into the right

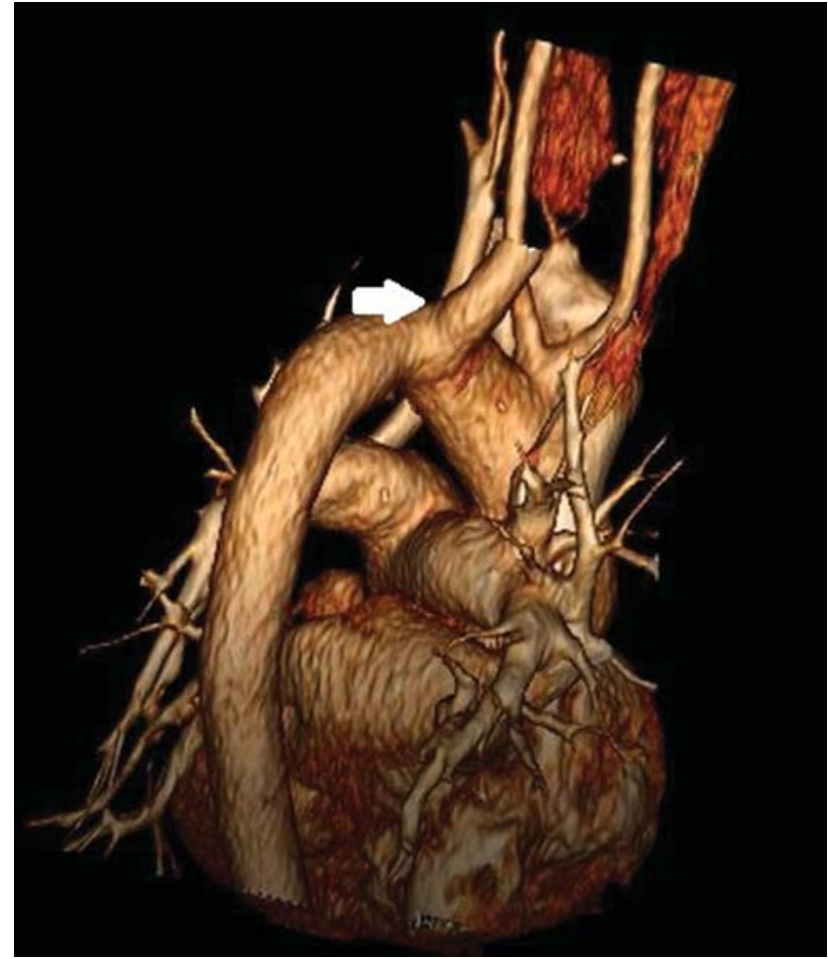

Fig. 3 Computed tomography of the thorax: three-dimensional reconstruction. The white arrow shows an aberrant right subclavian artery.

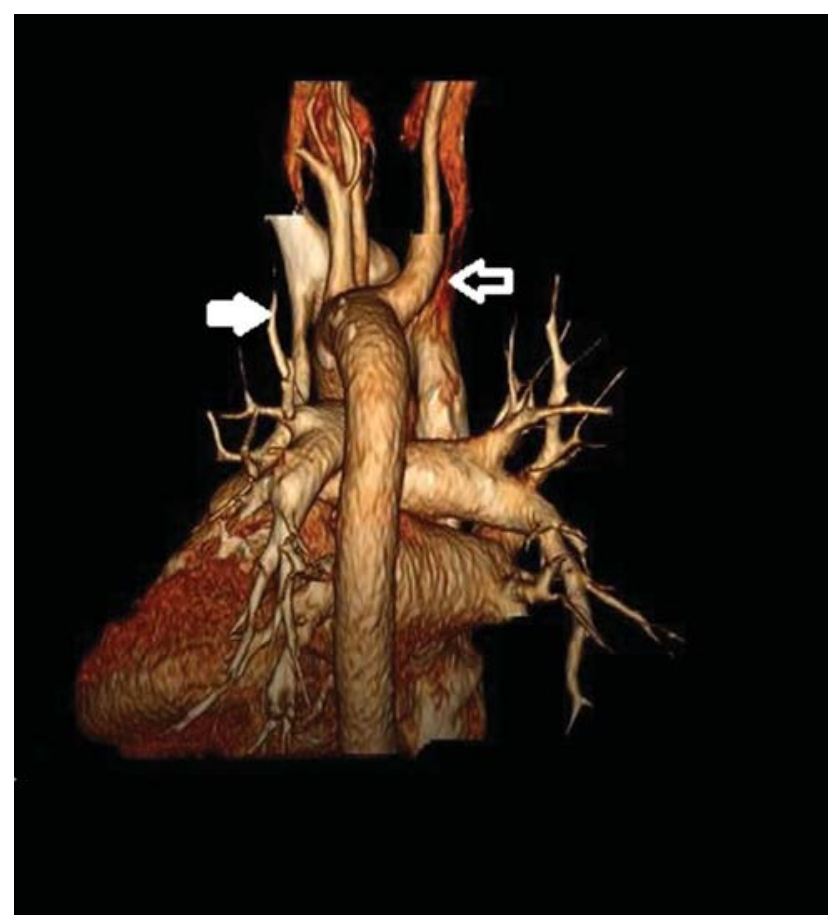

Fig. 4 Computed tomography of the thorax: three-dimensional reconstruction. The white arrow demonstrates the anomalous pulmonary vein of the left lung upper lobe, whereas the black and white arrow shows the aberrant right subclavian artery.

atrium, a central catheter inserted in this vessel has a functionally correct position.

Catheter placements in the internal thoracic vein or in the pericardiophrenic vein are rare but potentially dangerous. 
These are tiny thin-walled veins with a substantially high risk of perforation. Such a perforation could lead to a left-sided hemo- or infusothorax. Pericardial tamponade has also been described after the perforation of the pericardiophrenic vein, though it is quite hard to explain as this vein runs extrapericardial. ${ }^{4}$

Another possibility of a left thoracic intravascular placement of a venous catheter is, as described in our case, within an abnormally draining pulmonary vein. A partial anomalous pulmonary venous return has a prevalence of 0.4 to $0.7 \%$ within the population and is characterized by drainage of some but not all of the pulmonary veins into either the systemic veins or into the right atrium. The right lung is more commonly affected (80-90\%), whereas an atrial septal defect (ASD) of the sinus venosus type often accompanies this condition. A special form of a pulmonary vein connection to the inferior vena cava is called Scimitar's syndrome. ${ }^{5}$

Anomalous venous connections of the left lung affect either all of the veins of the left lung or only the upper lobe vein. In most cases, they flow into the innominate vein; in rare cases, they drain into the coronary sinus, into a left persistent vena cava superior or directly into the right atrium. ${ }^{6}$

In the case of our patient, the pulmonary vein of the left upper lobe drained into the innominate vein. The malposition of the venous catheter in the pulmonary vein is provable by monitoring of pressures with the help of the Swan-Ganz catheter. It was also possible to quantify the left-to-right shunt through the incorrectly attaching pulmonary vein. The oxygen saturation in blood measured through the left-sided catheter showed values consistent with arterial blood, whereas the medium pressures measured through both catheters were nearly equal. The slightly increased pressure in the pulmonary vein is explainable through the physiology of the central intrathoracic venous return. ${ }^{7}$ In addition, the mixed venous oxygen saturation was significantly lower than the central venous one, which indicates the presence of a leftto right-shunt. This was calculated as $\mathrm{Qp} / \mathrm{Qs}=1.36 / 1$.

An isolated incorrectly attaching pulmonary vein of the left upper lobe without accompanying ASD is not an indication for operation when Qp/Qs is less than $1.8 / 1$, as the shunt volume is not expected to increase with age. If a higher shunt volume is present, then the therapy of choice is transection of the anomalous vertical vein and anastomosis to the left atrial appendage. ${ }^{8}$ In rare cases, a lobectomy can also be indicated. ${ }^{5}$

In the case of our patient, we had an older contrast CT of the thorax in which the partial anomalous venous drainage could not be diagnosed at first but could be detected afterward (-Figs. 2-4).

Additionally, we had a correctly placed Swan-Ganz catheter and a catheter in the radial artery. This made it possible to diagnose the partial anomalous venous drainage and the shunt calculation and to exclude an operational indication. The left-sided catheter was easily removed without any complications.

Some authors report the usage of vein catheters in abnormally draining pulmonary veins for venous access or even hemodialysis for several days without any complications. ${ }^{9,10}$

In summary, the aspiration of arterialized blood from a venous catheter, which has been subjected to a pressure measurement and which seems to lie within the central venous system, should indicate the presence of a left-to-right shunt.

Conflict of Interest

None.

\section{References}

1 Ho ML, Bhalla S, Bierhals A, Gutierrez F. MDCT of partial anomalous pulmonary venous return (PAPVR) in adults. J Thorac Imaging 2009;24(02):89-95

2 Mikubo M, Ikeda S, Hoshino T, Yokota T, Fujii A, Mori M. Pulmonary resection of lung cancer in a patient with partial anomalous pulmonary venous connection. Ann Thorac Surg 2013;95(05): 1799-1801

3 Irwin RB, Greaves M, Schmitt M. Left superior vena cava: revisited. Eur Heart J Cardiovasc Imaging 2012;13(04):284-291

4 Wang L, Liu ZS, Wang CA. Malposition of central venous catheter: presentation and management. Chin Med J (Engl) 2016;129(02): 227-234

5 Nicholas Kouchoukos Eugene Blackstone Frank Hanley James Kirklin. Total anomalous pulmonary venous connection. Kirklin/Barratt-Boyes Cardiac Surgery. 4th ed. 2012:1182

6 Brody H. Drainage of the pulmonary veins into the right side of the heart. Arch Pathol Lab Med 1942;33:221-240

7 Berlin DA, Bakker J. Starling curves and central venous pressure. Crit Care 2015;19:55

8 ElBardissi AW, Dearani JA, Suri RM, Danielson GK. Left-sided partial anomalous pulmonary venous connections. Ann Thorac Surg 2008;85(03):1007-1014

9 Khanna AK, Maheshwari A, Popovich MJ, Mathur P. Incidental discovery of a partial anomalous pulmonary venous connection in the surgical critical care unit. J Clin Anesth 2014;26(08): 693-696

10 Grillot N, Figueiredo S, Aubry A, Leblanc PE, Duranteau J. Unusual dialysis catheter position due to partial anomalous pulmonary venous return: Diagnosis and management. Anaesth Crit Care Pain Med 2016;35(03):233-235 